\title{
A special kind of Optimal PID Control Method for High Speed Aircraft Attack Angle Tracking
}

\author{
Hui Wang ${ }^{1, a}$ and Junwei Lei ${ }^{2, b}$ \\ ${ }^{1}$ Naval Equipment Department of PLA, Equipment Purchase Center, Beijing, China \\ ${ }^{2}$ Department of control engineering, Naval Aeronautical and Astronautical University, Yantai, \\ 264001
}

awanghui1024@126.com, 'leijunwei@126.com

Keywords: hypersonic aircraft; stability; PID control; optimal control; numerical simulation

\begin{abstract}
A new kind of optimal PID control method was proposed to solve the attack angle tracking problem of hypersonic aircraft . Both the control of attack angle and speed of aircraft are realized by the optimal PID control strategy. And a kind of integration of error method was used to mark the performance of system with different setting of PID parameters, also the adjustment rule is divided into two kinds of situation to guarantee the convergence of adjustment of PID parameters. At last, detailed numerical simulation is done to testify the rightness of the proposed method.
\end{abstract}

\section{Introduction}

The pitch channel control problem, especially the attack angle tracking problem of hypersonic vehicle has aroused the interest of researchers in the world. Because of its strong nonlinear, fast time-varying characteristics and the three channel coupling, the research of the current control theory and control method has faced great challenges[1-5]. At present, many methods have been tried to control the hypersonic aircraft, including adaptive, variable structure control, also contains neural network and many other intelligent methods[6-8]. However, PID control method is still the most widely used method which has good robustness and reliability. But how to choose a group of PID parameters to make the system with satisfactory performance is still a worldwide difficult problem. In the past, PID parameters was set mostly by trial and error method. But it is very waste time. In this paper, a kind of optimal method was proposed to solve this problem, and the whole process can be finished by computer program. So it can save a lot of time and labor work.

\section{Model Description}

The pitch channel model of a kind of hypersonic missile can be written as:

$$
\begin{gathered}
\dot{V}=\frac{T \cos \alpha-D}{m}-g \sin \gamma \\
\dot{\gamma}=\frac{L+T \sin \alpha}{m V}-\frac{g \cos \gamma}{V} \\
\dot{\alpha}=q-\dot{\gamma} \\
\dot{q}=\frac{M}{I} \\
\dot{h}=V \sin \gamma \\
\ddot{\eta}=-2 \varsigma \omega_{n} \dot{\eta}-\omega_{n}^{2} \eta+\omega_{n}^{2} \eta_{c}, \omega_{n}=5, \varsigma=0.7
\end{gathered}
$$

Where parameters are defined as follows:

$$
\begin{aligned}
& L=\bar{q} S C_{L}, C_{L}=C_{L}^{\alpha} \alpha+C_{L}^{\delta} \delta+C_{L}^{0}, \quad T=\bar{q} S C_{T} \\
& D=\bar{q} S C_{D}, C_{D}=C_{d}^{\alpha^{2}} \alpha^{2}+C_{D}^{\alpha} \alpha+C_{D}^{\delta^{2}} \delta^{2}+C_{D}^{\delta} \delta+C_{D}^{0} \\
& M=\bar{q} S \bar{c}\left[C_{M \alpha}+C_{M \delta}+C_{M q}\right], C_{M \alpha}=C_{M \alpha}^{\alpha^{2}} \alpha^{2}+C_{M \alpha}^{\alpha} \alpha+C_{M \alpha}^{0},
\end{aligned}
$$




$$
\begin{aligned}
& C_{M \delta}=C_{e}\left(\delta_{e}-\alpha\right) \\
& C_{M \alpha}=10^{-4}\left(0.06-e^{-M_{a} / 3}\right)\left(-2 \alpha^{2}+120 \alpha-1\right) \\
& C_{M q}=\frac{\bar{c} q}{2 V}\left(-0.025 M_{a}+1.37\right)\left(-0.0021 \alpha^{2}+0.0053 \alpha-0.23\right) \\
& C_{M \delta}=0.0292(\delta-\alpha) \\
& C_{L}=\alpha\left(0.493+1.91 / M_{a}\right) \\
& C_{D}=0.0082\left(171 \alpha^{2}+1.15 \alpha+2\right)\left(0.0012 M_{a}^{2}-0.054 M_{a}+1\right) \\
& C_{T}=\left\{\begin{array}{l}
38\left[1-164\left(\alpha-\alpha_{0}\right)^{2}\right]\left(1+17 / M_{a}\right)(1+0.15) \eta, \eta<1 \\
38\left[1-164\left(\alpha-\alpha_{0}\right)^{2}\right]\left(1+17 / M_{a}\right)(1+0.15 \eta), \eta<1
\end{array}\right\}
\end{aligned}
$$

And $V$ is speed, $\gamma$ is the speed angle, $\alpha$ is attack angle, $Q$ is the attitude angle speed, $h$ is the height. $\phi$ is the oil supplying factor, $\delta_{c}$ is the duck wing and $\delta_{e}$ is the lift rudder.

\section{Optical PID Controller Design for Tracking of Attack Angle}

Firstly, design the PID controller for the attack angle tracking system of hypersonic aircraft as follows:

$$
\begin{gathered}
e_{\alpha}=\alpha-\alpha^{d} \\
\delta=k_{p} e_{\alpha}+k_{i} \int e_{\alpha} d t+k_{d} \dot{e}_{\alpha}+k_{q} q
\end{gathered}
$$

Where $\alpha^{d}$ is the desired attack angle, and $e_{\alpha}$ is the error of attack angle.

Second, design the PID controller for the speed tracking system of hypersonic aircraft as follows:

$$
\begin{gathered}
e_{v}=V^{d}-V \\
\begin{cases}\phi_{c}=k_{p} e_{v}+k_{s} \int e_{v} d t+k_{d} \dot{e}_{v} & e_{v}>0 \\
\phi_{c}=-30 & e_{v}<0\end{cases}
\end{gathered}
$$

Where $V^{d}$ is the desired speed of aircraft, and $e_{v}$ is the error of speed.

At last, design the optimal principle for the parameter adjustment of above PID controllers as follows. Here we use the integration of the absolute value of error to describe the performance of the system.

$$
\text { area }=\int_{0}^{t}|e(t)| d t
$$

Where define score as follows to mark every system with different PID parameters.

$$
\text { score }=\frac{1}{\text { area }}
$$

Where the main structure of adjustment of optimal PID parameters can be described as bellow figures.

So if the score is very big, it means that the PID parameter is better than previous one. Where the adjustment for the PID parameters can be described as below equations:

$$
k_{p}(j)=k_{p}(j-1)+a
$$

If $\operatorname{score}(j-1)<29.0$, then $a$ can be computed as

$$
a=0.99 \operatorname{sign}\left(\frac{\operatorname{score}(j-1)-\operatorname{score}(j-2)}{k_{p}(j-1)-k_{p}(j-2)}\right)
$$

If $\operatorname{score}(j-1)>29.0$, then $a$ can be computed as 


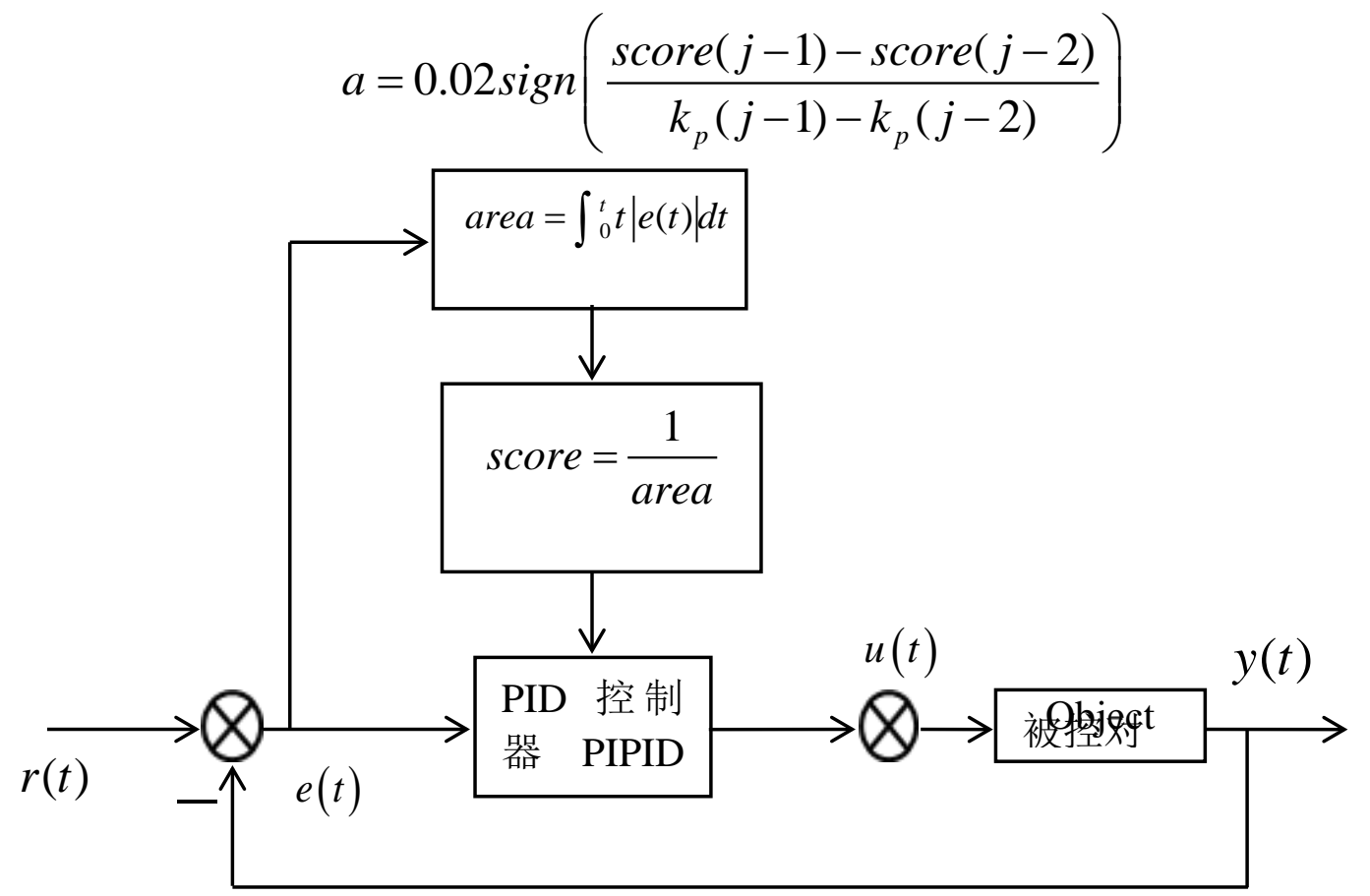

Figure 1 The structure of adjustment of optimal PID parameter

\section{Numerical Simulation and Result Analysis}

Set model parameters as

$$
\begin{aligned}
& I_{x x}=-7.1 * 10^{-5} \mathrm{~m}^{2}+19.1 \mathrm{~m}-59430 \\
& I_{y y}=-8.03 * 10^{-4} \mathrm{~m}^{2}+219.74 m-1690000 \\
& I_{z z}=-8.03 * 10^{-4} \mathrm{~m}^{2}+219.74 m-1690000 \\
& I_{y y 0}=1.23 * 10^{7}, \quad v_{s}=3.017 * 10^{2}, \rho_{a}=1.84 * 10^{2}, \\
& g_{a}=9.7147, \quad h=30000, V=4525, \eta_{c}=0.15662
\end{aligned}
$$

With above PID controller and parameter adjustment method, the response of attack angle with PID controller can see below figure 2, where desired value of attack angle is set as 4 degree, and simulation results can be shown as following figures 2 to figures 5 .

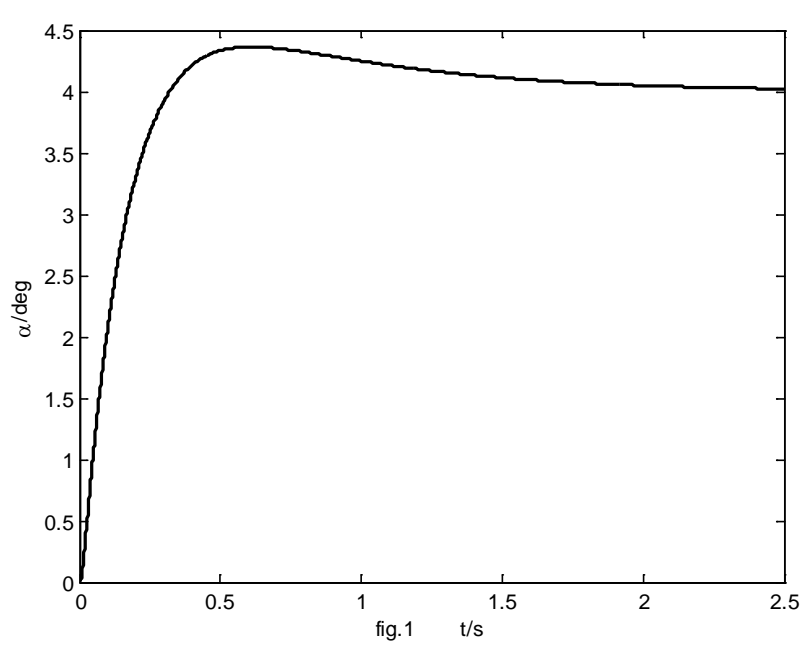

Figure 2 The curve of attack angle

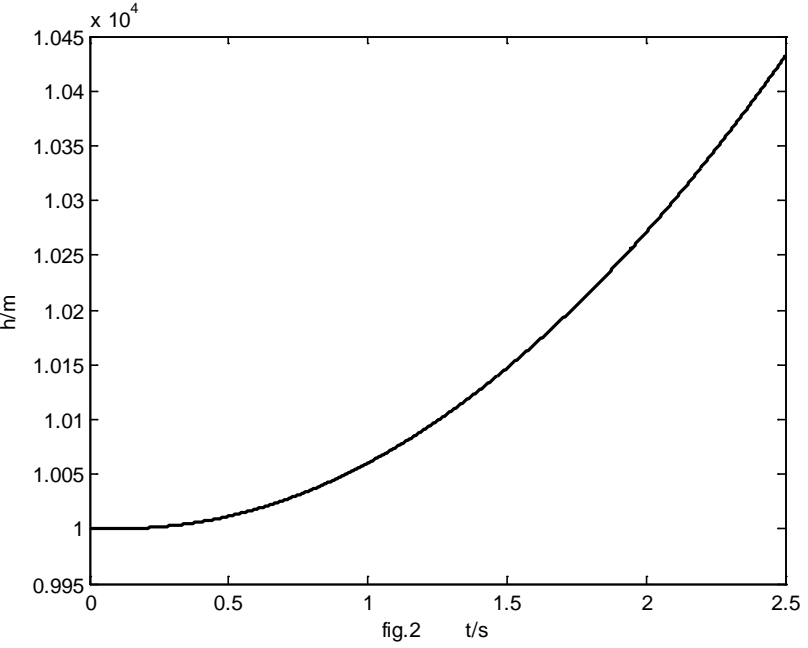

Figure 3 The curve of height 


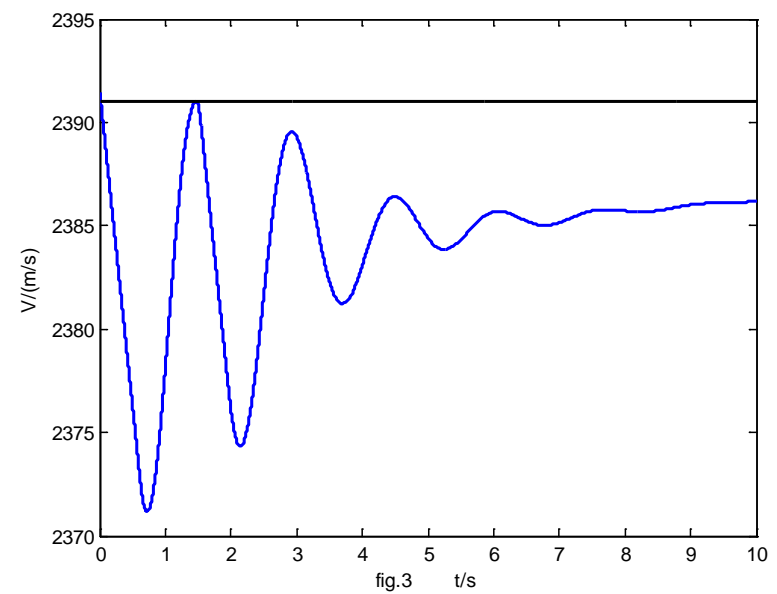

Figure 4 The curve of speed

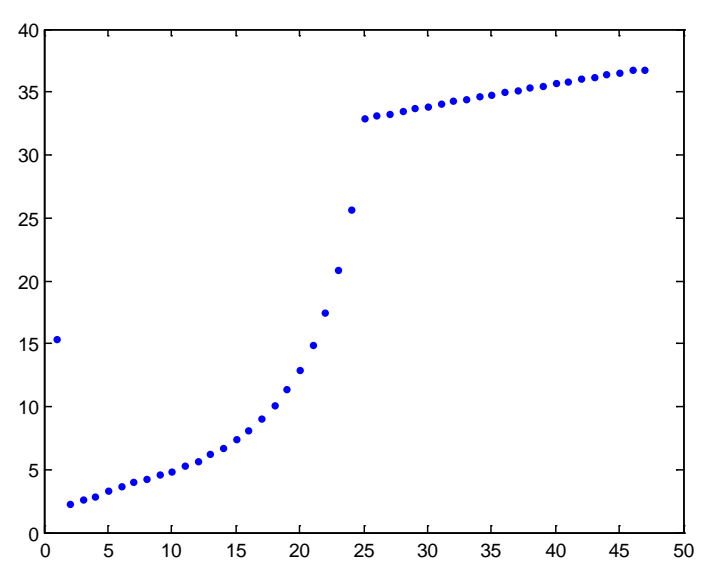

Figure 6 The curve of score

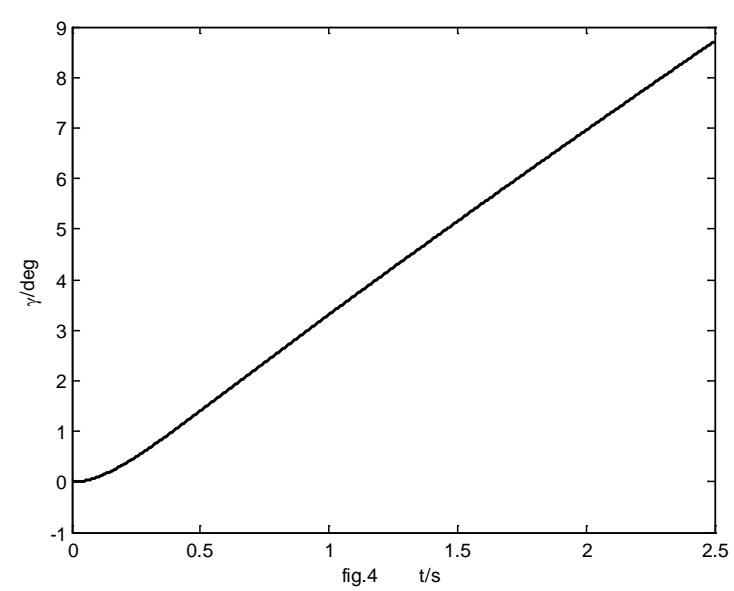

Figure 5 The curve of speed angle

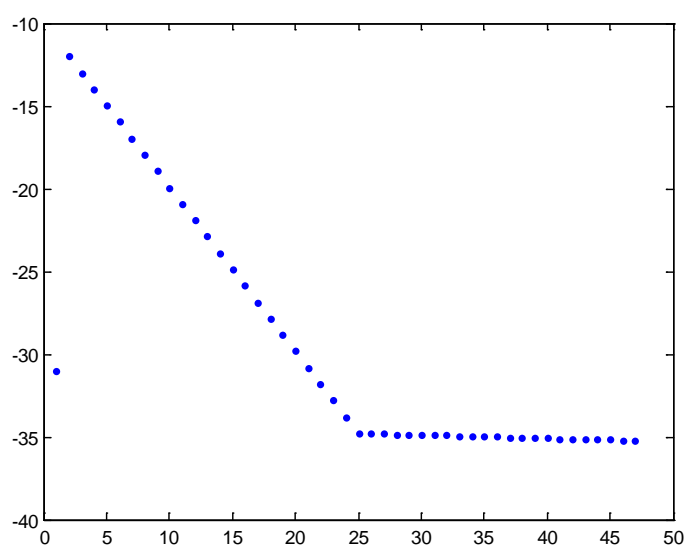

Figure 7 The curve of $\mathrm{P}$ parameter

We can find the score is rising with the proposed optimal method to adjust PID parameters and the whole simulation times is set as 100 , and the optimal value was found at the 47th simulation.

\section{Conclusion}

First, PID controllers were designed to control the attack angle and speed of pitch channel dynamics of hypersonic aircraft. And the model was nonlinear with strong uncertainties. Second, a kind of novel optimal method was proposed to solve the parameter adjustment of PID controller. And the performance of the system was described by marking a score method with integration of absolute value of error. Also, two kinds of parameter adjustment law was proposed to make the parameter converge to the optimal value. At last, 100 simulations were done to testify the rightness of the proposed method.

\section{References}

[1]Buschek $\mathrm{H}$ and Calise A J. Hypersonic flight control system design using fixed order robust controllers[A]. In:AIAA Guidance, Navigation and Control Conference[C]. Chattanooga, TN, AIAA 95-6062

[2]Buschek H, Calise A J. Uncertainty modeling and fixed-order controller design for a hypersonicvehicle model. Journal of Guidance, Control and Dynamics, 1997, 20(1): 42-48.

[3]Heller $M$ and Sachs G.Flight dynamics and robust control of a hypersonic test vehicle with ramjet propulsion[A]. In:AIAA International Space Planes and Hypersonic Systems and Technologies Conference [C].Norfolk,VA,AIAA 98-1521 
[4]M. Ohno, Y. Yamaguchi, T. Hata, M. Takahama, Robust flight control law design for an automatic landing flight experiment [J], Control Engineering Practice, 1999, 7(9):1143-1152

[5] Sergio B.Varella Gomes and Josue Jr.G.Ramos. Airship dynamic modeling for autonomous operation. Proceedings of the 2003 IEEE. International Conference on Robotics \& Automation. 2003:5 14

[6] J.S.Uhlman, N.E.Fine, D.C.Kring. Calculation of the Added Mass and Damping Forces on Supercavitating Bodies. The 4th International Symposium on Cavitation,California,2001:7 13

[7] D.Clarke.Calculation of the Added Mass of Elliptical Cylinders in Shallow Water. Ocean Engineering. 2001, 28(4):61 72

[8] C.J.Atkinson,R.G.Urso. Modeling of Apparent Mass Effects for the Real-Time Simulation of a Hybird Airship.AIAA Modeling and Simulation Technologies Conference and Exhibit, Keystone. 2006:21 32 